\title{
La investigación sobre Historia de la Comunicación en España: el caso de las revistas fuente de IN-RECS (2002-2012)
}

\author{
Ricardo ZugASti Azagra \\ Universidad San Jorge (Zaragoza) \\ rzugasti@usj.es
}

\begin{abstract}
Resumen
En España se ha empezado recientemente a abordar la investigación sobre la propia investigación que se hace en el ámbito de la Comunicación. Sin embargo, en el campo más concreto de la Historia de la Comunicación existe una carencia de este tipo de estudios. Este trabajo busca cubrir en parte esa escasez, investigando las características de la investigación actual sobre Historia de la Comunicación. Para ello se aplicó un análisis de contenido cuantitativo a todos los artículos sobre esta disciplina publicados entre 2002 y 2012 en las revistas fuente del índice bibliométrico IN-RECS.
\end{abstract}

Palabras clave: investigación en Comunicación; Historia de la Comunicación; revistas científicas.

Research about Communication History in Spain: The case of the most influential journals included in the bibliometric index IN-RECS (2002-2012)

\begin{abstract}
The research about the research done in Communication has been tackled in Spain in recent time. However, there is a lack of this kind of research in the specific area of Communication History. This piece of research aims to offer one of the first studies in this field, focused in the main characteristics of current research in Communication History. A quantitative content analysis was applied to every article published between 2002 and 2012 in the most influential IN-RECS-index journals.
\end{abstract}

Key Words: Communication research; Communication History; scientific journals.

\section{Referencia normalizada:}

Zugasti Azagra, R. (2013) La investigación sobre Historia de la Comunicación en España: el caso de las revistas fuente de IN-RECS (2002-2012). Historia y Comunicación Social. Vol. 18. № Especial Diciembre. Págs. 65-76.

Sumario: 1. Introducción. 2. Metodología. 3. Resultados. 4. Conclusiones. 5. Bibliografía.

\section{Introducción}

El auge que la investigación científica sobre Comunicación ha experimentado en España durante, aproximadamente, la última década es llamativo. La multiplicación de universidades que ofertan titulaciones de Comunicación, más de 45 en la actuali- 
dad, ha contribuido de manera poderosa al desarrollo de una disciplina que llegó no hace mucho a la universidad de manera oficial: en 1971, con las Facultades de Ciencias de la Información de las universidades Complutense de Madrid, Autónoma de Barcelona y Navarra (Vigil y Vázquez, 1987: 153-265). Además, las nuevas exigencias de obtener resultados de investigación para promocionar en la carrera académica han provocado una dinamización de la actividad investigadora, también en el ámbito de la Comunicación. Ha sido una de las consecuencias de lo que Soriano ha bautizado gráficamente como "efecto ANECA" (2008: 118).

La investigación en Comunicación está abordando como objeto de estudio, cada vez con mayor ímpetu, la propia investigación en este campo, en un afán por conocer sus peculiaridades, intereses, carencias, sistemas de difusión, etc. Es un síntoma, como señalan Martínez Nicolás y Saperas Lapiedra, de la "madurez" del ámbito científico de la comunicación (2011: 106). Trabajos como los de Moragas en la década de 1980, que abren esta línea en España (1988), los de Jones (1998), o los más recientes de Xifra y Castillo (2006), Martínez Nicolás (2008), Colle (2009), Castillo y Carretón (2010), Castillo, Rubio y Almansa (2012), De Filippo (2013), Escribá y Cortiñas (2013) son ejemplos destacados de esta nueva línea investigadora en el campo de la Comunicación.

Sin embargo, este investigar sobre la investigación no ha profundizado todavía en el estudio de cada una de las distintas disciplinas de la Comunicación. Es en este punto donde el presente trabajo pretende contribuir a través de un análisis de la investigación en Historia de la Comunicación. Esta disciplina está presente, de una manera u otra, en la práctica totalidad de los planes de estudio vigentes en España; cuenta con la Asociación de Historiadores de la Comunicación desde 1992; se publican en España dos revistas científicas con este enfoque, Historia y Comunicación Social y Revista Internacional de Historia de la Comunicación. Además, muestra del peso de la disciplina son los trabajos que, acerca de la propia naturaleza de esta, han publicado Ruiz Acosta (1997), Román Portas (2000), Montero Díaz y Rueda Laffond (2001), Checa Godoy (2008) y Gómez Mompart (2008). Pese a este desarrollo de la Historia de la Comunicación, tan sólo ha visto la luz un trabajo que analiza empíricamente la investigación en este campo: un estudio temático de los artículos de Historia del Periodismo publicados en una selección de revistas de Comunicación incluidas en la base de datos DICE (De Haro y Martínez, 2011).

\section{Metodología}

El objetivo general de este trabajo es analizar la investigación sobre Historia de la Comunicación publicada recientemente como artículo en una selección de revistas científicas españolas de Comunicación. Las revistas escogidas son las consideradas como fuente de la disciplina de Comunicación en el índice bibliométrico IN-RECS, que calcula anualmente el índice de impacto de las revistas españolas de Ciencias Sociales. La muestra constituye por ello el núcleo de revistas científicas de Comu- 
nicación publicadas en España más influyentes: Anàlisi, Comunicación y Sociedad, Comunicar, Estudios sobre el Mensaje Periodístico, Revista Latina de Comunicación Social, Telos y ZER. El periodo analizado comprende la última década, de 2003 a 2012.

La metodología empleada es el análisis de contenido cuantitativo, con un código de elaboración propia que se aplica a cada artículo sobre Historia de la Comunicación publicado por estas revistas en el periodo señalado. El código contempla una serie de variables para obtener datos estadísticos acerca de algunos rasgos de los artículos que conforman la muestra: el año de publicación, el ámbito de la Comunicación al que pertenece, el medio o soporte del que trata, el elemento del proceso comunicativo en el que se centra, el siglo del objeto de estudio, el origen geográfico del tema, si emplea una metodología cuantitativa o cualitativa, así como sobre el número y características de los autores. A través del empleo de tablas de contingencia se pueden asimismo comparar los resultados obtenidos entre las diferentes revistas.

\section{Resultados}

En total, fueron revisados 2.489 artículos, esto es, la totalidad de los publicados por las siete revistas objeto de análisis entre los años 2003 y 2012. De todos ellos, 149 pertenecen al ámbito de la Historia de la Comunicación, lo que supone el 6\% del total. Distinguiendo entre títulos, como muestra la tabla "Distribución de artículos por revista", Estudios sobre el Mensaje Periodístico es la revista que, porcentualmente, más cabida da entre sus páginas a artículos sobre Historia de la Comunicación, con casi el 14\% del total. Le siguen ZER (10,1\%), Comunicación y Sociedad (9,5\%), Anàlisi (9,3\%) y, algo más distanciada, Revista Latina de Comunicación Social (5,2\%). El escaso peso de la disciplina en Telos (3,9\%) y Comunicar (1\%) se debe al perfil especializado de ambas, que dificulta la inclusión de trabajos de contenido histórico. La publicación de la Fundación Telefónica está enfocada a las Tecnologías de la Información y la Comunicación. Comunicar, por su parte, presenta un contenido centrado fundamentalmente en la intersección entre Comunicación y Educación.

a) Distribución de artículos por revista

\begin{tabular}{|l|c|c|c|}
\hline \multicolumn{1}{|c|}{ Revista } & Total artículos & $\begin{array}{c}\text { Artículos Historia de la } \\
\text { Comunicación }\end{array}$ & Porcentaje sobre el total \\
\hline Análisi & 182 & 17 & 9,3 \\
\hline CyS & 168 & 16 & 9,5 \\
\hline Comunicar & 669 & 7 & 1 \\
\hline EMP & 309 & 43 & 13,9 \\
\hline RLCS & 347 & 18 & 5,2 \\
\hline Telos & 547 & 21 & 3,8 \\
\hline ZER & 267 & 27 & 10,1 \\
\hline
\end{tabular}




\begin{tabular}{|l|l|l|l|}
\hline Total & 2.489 & 149 & 6 \\
\hline
\end{tabular}

Tomando en cuenta sólo los datos absolutos, plasmados en la tabla "Distribución de artículos sobre Historia de la Comunicación por revista", resulta que Estudios sobre el Mensaje Periodístico es la que más artículos sobre Historia de la Comunicación incluye (el 28,9\%), frente a Comunicar, la que menos con un 4,7\%.

b) Distribución de artículos sobre Historia de la Comunicación por revista

\begin{tabular}{|l|c|c|}
\hline \multicolumn{1}{|c|}{ Revista } & Número & Porcentaje \\
\hline Análisi & 17 & 11,4 \\
\hline CyS & 16 & 10,7 \\
\hline Comunicar & 7 & 4,7 \\
\hline EMP & 43 & 28,9 \\
\hline RLCS & 18 & 12,1 \\
\hline Telos & 21 & 14,1 \\
\hline ZER & 27 & 18,1 \\
\hline Total & $\mathbf{1 4 9}$ & $\mathbf{1 0 0}$ \\
\hline
\end{tabular}

En cuanto a la distribución cronológica de los artículos publicados por el conjunto de las revistas, no se observan variaciones llamativas. La menor frecuencia de publicación se da en 2004 con 9 artículos, y la mayor se produce en 2010 con 23 artículos. La gráfica "Artículos por año" muestra la evolución a lo largo de la década analizada.

c) Artículos por año

Los datos sobre el ámbito del que tratan los artículos son reveladores del peso sustancial del Periodismo frente a otras áreas, lo que está en consonancia con las conclusiones de otros trabajos referidos al ámbito de la Comunicación en general (Martínez Nicolás y Saperas Lapiedra, 2011: 114). Así, el 53\% del total de artículos versan sobre Periodismo. El segundo ámbito más tratado es la Comunicación Audiovisual, con un notable 27,5\%. A distancia les sigue la Propaganda, con un $12,1 \%$. Llama la atención el mínimo porcentaje de trabajos centrados en la Publicidad $(2,7 \%)$ y las Relaciones Públicas (2\%). Los artículos que versan sobre varios ámbitos de los anteriores alcanzan un escaso $2,7 \%$.

Según indican los datos expuestos en la tabla "Ámbito del artículo por revista", el Periodismo es el área más tratada en todas las publicaciones, con la excepción de Comunicar y de Telos, en las que domina la Comunicación Audiovisual (57,1\% y $47,6 \%$ respectivamente). El hecho de que la primera esté especializada en temas de educomunicación, donde las pantallas tienen una especial relevancia, y de que la segunda lo esté en las TIC explican estos resultados. Del mismo modo, la especialización de Estudios sobre el Mensaje Periodístico en investigaciones sobre Periodismo hace que ostente el porcentaje más alto de artículos sobre este ámbito (74,4\%). 
d) Ámbito del artículo por revista (en porcentaje)

\begin{tabular}{|c|c|c|c|c|c|c|c|}
\hline Ámbito & Anàlisi & $C y S$ & Com. & $E M P$ & RLCS & Telos & ZER \\
\hline Periodismo & 41,2 & 43,8 & 14,3 & 74,4 & 72,2 & 38,1 & 40,7 \\
\hline Publicidad & 0 & 0 & 0 & 0 & 11,1 & 9,5 & 0 \\
\hline RR.PP. & 11,8 & 0 & 0 & 0 & 0 & 0 & 3,7 \\
\hline Propaganda & 17,6 & 6,3 & 14,3 & 9,3 & 11,1 & 0 & 25,9 \\
\hline Com. Aud. & 23,5 & 50 & 57,1 & 16,3 & 5,6 & 47,6 & 25,9 \\
\hline Varios & 5,9 & 0 & 14,3 & 0 & 0 & 4,8 & 3,7 \\
\hline Total & 100 & 100 & 100 & 100 & 100 & 100 & 100 \\
\hline
\end{tabular}

Por lo que respecta al medio o soporte en el que se centra el artículo, la prensa escrita es la estrella: más de la mitad de las investigaciones publicadas se centran en ella (50,3\%). Como indica Checa Godoy, "la historia de la prensa goza de una tradición, y en cierta medida de un reconocimiento, de los que carece la historia de otros medios de comunicación, sin más excepción que la del cine" (2008: 71). El segundo puesto lo ocupa, consecuente con la cita anterior, el cine, que aglutina el 13,4\% de los trabajos. La televisión y la radio concentran el $11,4 \%$ y el $8,1 \%$ respectivamente. Menos atención suscitan la cartelería e Internet, con un 1,3\% la primera y un 3,4\% el segundo. Con respecto a Internet, Díaz Noci apunta a que muchos historiadores son reticentes a estudiar periodos inacabados (2013: 255). Por último, un 8,1\% de los textos tratan sobre varios de los medios anteriores.

e) Medio objeto de estudio por revista (en porcentaje)

\begin{tabular}{|c|c|c|c|c|c|c|c|}
\hline Medio & Anàlisi & $C y S$ & Com. & $E M P$ & $R L C S$ & Telos & ZER \\
\hline Prensa & 52,9 & 43,8 & 0 & 79,1 & 72,2 & 9,5 & 37 \\
\hline Radio & 11,8 & 0 & 0 & 9,3 & 0 & 9,5 & 14,8 \\
\hline TV & 11,8 & 25 & 14,3 & 2,3 & 5,6 & 33,3 & 3,7 \\
\hline Cine & 5,9 & 31,3 & 42,9 & 4,7 & 11,1 & 9,5 & 18,5 \\
\hline Cartel & 0 & 0 & 0 & 0 & 5,6 & 4,8 & 0 \\
\hline Internet & 0 & 0 & 0 & 2,3 & 5,6 & 14,3 & 0 \\
\hline Varios & 5,9 & 0 & 42,9 & 0 & 0 & 19 & 14,8 \\
\hline Otros & 11,8 & 0 & 0 & 2,3 & 0 & 0 & 11,1 \\
\hline Total & 100 & 100 & 100 & 100 & 100 & 100 & 100 \\
\hline
\end{tabular}

El elemento del proceso comunicativo que más relevancia adquiere es el análisis de los mensajes (37,6\%). En este sentido, las investigaciones sobre el contenido de los medios son mayoritarias en Comunicación, como demuestra también el trabajo de Martínez Nicolás y Saperas Lapiedra (2011: 116). Un 26,8\% de los artículos investigan sobre los emisores, y tan solo el $4 \%$ lo hacen sobre los receptores. Un considerable $31,5 \%$ de los trabajos se circunscriben a dos o más de los elementos anteriores. La tabla "Elemento de la comunicación objeto del artículo por revista" muestra que 
Comunicar es la única revista que prioriza a los receptores, lo que concuerda con su carácter especializado en Comunicación y Educación.

f) Elemento de la comunicación objeto del artículo por revista (en porcentaje)

\begin{tabular}{|c|c|c|c|c|c|c|c|}
\hline Elemento & Anàlisi & CyS & Com. & EMP & RLCS & Telos & ZER \\
\hline Emisor & 41,2 & 25 & 14,3 & 30,2 & 11,1 & 38,1 & 18,5 \\
\hline Mensaje & 41,2 & 37,5 & 14,3 & 41,9 & 44,4 & 14,3 & 48,1 \\
\hline Receptor & 0 & 0 & 28,6 & 0 & 0 & 9,5 & 7,4 \\
\hline Varios & 17,6 & 37,5 & 42,9 & 27,9 & 27,9 & 38,1 & 25,9 \\
\hline Total & 100 & 100 & 100 & 100 & 100 & 100 & 100 \\
\hline
\end{tabular}

El siglo XX es el periodo en el que se ubica cronológicamente una abrumadora mayoría de trabajos. E1 73,8\% de los artículos historian el pasado siglo. Le sigue en importancia el siglo XIX, con un 7,4\% de los trabajos. Siglos más lejanos como el XVI, XVII y XVIII concentran muy poco porcentaje de investigaciones: 1,3\%, 2,7\% y $0,7 \%$ respectivamente. Estos resultados concuerdan con los obtenidos por De Haro y Martínez (2011: 232-233) y reafirman que "una mayoría aplastante de la Historia de la Comunicación se ubica en lo que convencionalmente llamamos historia contemporánea" (Checa Godoy, 2008: 41). Destaca asimismo que un poco más del 14\% de los artículos aborden más de un siglo, lo que apunta a investigaciones de carácter diacrónico. La tabla "Siglo del objeto de estudio por revista" ofrece la distribución de cada título por separado. En todas las revistas prima el siglo XX, y las únicas que publican artículos sobre los siglos XVI, XVII y XVIII son Anàlisi, Estudios sobre el Mensaje Periodístico y ZER.

g) Siglo del objeto de estudio por revista (en porcentaje)

\begin{tabular}{|c|c|c|c|c|c|c|c|}
\hline Siglo & Anàlisi & $C y S$ & Com. & EMP & RLCS & Telos & ZER \\
\hline XVI & 5,9 & 0 & 0 & 2,3 & 0 & 0 & 0 \\
\hline XVII & 0 & 0 & 0 & 7 & 0 & 0 & 3,7 \\
\hline XVIII & 0 & 0 & 0 & 2,3 & 0 & 0 & 0 \\
\hline XIX & 5,9 & 6,3 & 0 & 14 & 5,6 & 0 & 7,4 \\
\hline XX & 76,5 & 93,8 & 100 & 55,8 & 77,8 & 66,7 & 85,2 \\
\hline Varios & 11,8 & 0 & 0 & 18,6 & 16,7 & 33,3 & 3,7 \\
\hline Total & 100 & 100 & 100 & 100 & 100 & 100 & 100 \\
\hline
\end{tabular}

Si el siglo XX es el periodo estrella, atendiendo al ámbito geográfico al que se circunscribe el objeto de investigación de los artículos, el lugar estrella es España. El $62,4 \%$ del total de trabajos tratan temas que se desarrollan en el mismo país de edición que las revistas analizadas. El segundo lugar en importancia es Iberoamérica, con un 10,7\%. Otros países europeos concentran el 8,7\% y los Estados Unidos, el $4 \%$. Cabe destacar que un $12,8 \%$ de los artículos abordan más de un ámbito geográfico, lo que deja entrever trabajos de tipo comparativo. En la tabla "Origen geográfico del tema por revista" se observa que Telos es la única publicación en la que España no 
es el principal lugar, sino que son mayoría los artículos con asuntos plurilocalizados, y en la que Estados Unidos supera a Iberoamérica.

h) Origen geográfico del tema por revista (en porcentaje)

\begin{tabular}{|c|c|c|c|c|c|c|c|}
\hline Origen del tema & Anàlisi & $C y S$ & $C o m$. & $E M P$ & $R L C S$ & Telos & ZER \\
\hline España & 64,7 & 75 & 57,1 & 67,4 & 61,1 & 23,8 & 77,8 \\
\hline Otro país europeo & 5,9 & 12,5 & 14,3 & 9,3 & 5,6 & 9,5 & 7,4 \\
\hline EEUU & 0 & 0 & 0 & 2,3 & 0 & 19 & 3,7 \\
\hline Iberoamérica & 5,9 & 0 & 14,3 & 11,6 & 16,7 & 14,3 & 11,1 \\
\hline Otros & 0 & 0 & 14,3 & 2,3 & 0 & 0 & 0 \\
\hline Varios & 23,5 & 12,5 & 0 & 7 & 16,7 & 33,3 & 0 \\
\hline Total & 100 & 100 & 100 & 100 & 100 & 100 & 100 \\
\hline
\end{tabular}

En cuanto a la metodología de investigación, el enfoque cualitativo supera abrumadoramente al cuantitativo. E1 85,9\% de los artículos emplean técnicas cualitativas frente al escaso 5,4\% que recurren a las cuantitativas. Estos datos difieren de los obtenidos por Castillo y Carretón en su estudio de todos los trabajos publicados en las diez revistas con mayor factor de impacto en IN-RECS en 2008, en el que se muestra que la investigación es mayoritariamente cuantitativa (2010: 313). Parece que el predominio del enfoque cualitativo es, por lo tanto, un rasgo particular de la Historia de la Comunicación, debido quizá al empleo de metodologías propias de la ciencia histórica. Por otro lado, un $8,7 \%$ de las investigaciones emplean ambas perspectivas para abordar el mismo tema. La tabla "Metodología utilizada por revista" permite observar que las revistas donde más presencia tienen las investigaciones cuantitativas, bien en exclusiva bien en triangulación con las cualitativas, son Anàlisi, Comunicación y Sociedad, y Revista Latina de Comunicación Social.

i) Metodología utilizada por revista (en porcentaje)

\begin{tabular}{|c|c|c|c|c|c|c|c|}
\hline Metodología & Anàlisi & $C y S$ & Com. & $E M P$ & $R L C S$ & Telos & ZER \\
\hline Cuantitativa & 11,8 & 6,3 & 14,3 & 4,7 & 5,6 & 0 & 3,7 \\
\hline Cualitativa & 70,6 & 81,3 & 85,7 & 88,4 & 72,2 & 100 & 92,6 \\
\hline Ambas & 17,6 & 12,5 & 0 & 7 & 22,2 & 0 & 3,7 \\
\hline Total & 100 & 100 & 100 & 100 & 100 & 100 & 100 \\
\hline
\end{tabular}

Lo habitual en los artículos analizados es que la autoría sea individual: un 83,9\% están firmados por una sola persona. Un 10,7\% tienen dos autores y un 5,4\% poseen más de dos firmantes. Estos resultados están en línea con lo que es habitual en los artículos en el ámbito de la Comunicación (Escribá y Cortiñas, 2013: 42; Roca y Pueyo, 2012: 311). De la tabla "Número de autores de cada artículo por revista" se extrae que la excepción a esta tendencia de la firma individual o por parejas es la revista Comunicar, en la que un $28,6 \%$ de los textos tienen más de dos autores. 
Continuando con los resultados relativos a los autores, en el $48 \%$ de los casos en los que el artículo ostenta más de un firmante, estos pertenecen a la misma institución, normalmente una universidad, como se verá más adelante. En el otro 48\% los autores están vinculados a instituciones diferentes. Estos resultados respaldan conclusiones de otros trabajos que ponen de manifiesto la escasa colaboración entre universidades (Roca y Pueyo, 2012: 312).

j) Número de autores de cada artículo por revista (en porcentaje)

\begin{tabular}{|c|c|c|c|c|c|c|c|}
\hline Autores & Anàlisi & $C y S$ & Com. & EMP & RLCS & Telos & ZER \\
\hline Uno & 82,4 & 87,5 & 71,4 & 83,7 & 88,9 & 85,7 & 81,5 \\
\hline Dos & 11,8 & 12,5 & 0 & 14 & 5,6 & 14,3 & 7,4 \\
\hline Más de dos & 5,9 & 0 & 28,6 & 2,3 & 5,6 & 0 & 11,1 \\
\hline Total & 100 & 100 & 100 & 100 & 100 & 100 & 100 \\
\hline
\end{tabular}

La tabla "Los autores son profesores universitarios" muestra que el 94\% de los primeros firmantes son profesores universitarios, considerando como tales a los docentes universitarios de cualquier categoría así como a los becarios predoctorales. Tan sólo el 4,7\% no lo son. En el caso de los segundos autores disminuye al 81,8\% el porcentaje de profesores y aumenta hasta el 13,6\% el de quienes no lo son. Son, en todo caso, datos coherentes con los obtenidos por otros trabajos donde se muestra el aplastante dominio de los autores académicos (Martínez Nicolás y Saperas Lapiedra, 2011: 111; Rubio y Almansa, 2012: 267; Escribá y Cortiñas, 2013: 40).

k) Los autores son profesores universitarios (en porcentaje)

\begin{tabular}{|c|c|c|}
\hline Profesor universitario & Primer autor & Segundo autor \\
\hline Sí & 94 & 81,8 \\
\hline No & 4,7 & 13,6 \\
\hline No se indica & 1,3 & 4,5 \\
\hline Total & 100 & 100 \\
\hline
\end{tabular}

Cuando los primeros autores son profesores universitarios, el 85,7\% pertenecen a una universidad española, frente al 14,3\% que están vinculados con una universidad extranjera, según se desprende de la tabla "Ubicación de la universidad de los autores". Con los segundos firmantes los datos son similares: el 83,3\% son docentes en centros españoles y el 16,7\% en extranjeros. La internacionalización de las contribuciones es escasa, en línea con los resultados de otros estudios referidos a la investigación en Comunicación en general (Escribá y Cortiñas, 2013: 42).

1) Ubicación de la universidad de los autores (en porcentaje)

\begin{tabular}{|c|c|c|}
\hline Ubicación universidad & Primer autor & Segundo autor \\
\hline España & 85,7 & 83,3 \\
\hline Extranjero & 14,3 & 16,7 \\
\hline Total & 100 & 100 \\
\hline
\end{tabular}


Si se consideran los autores únicos o los primeros firmantes en caso de que haya más de uno, destaca la Universidad Complutense de Madrid como la de mayor producción, con 21 artículos que suponen el 14\% del total. En la tabla "Las diez universidades con más artículos" se ofrecen los datos particulares de los centros que más artículos publican. Como se observa, todas son universidades españolas: Complutense de Madrid, País Vasco, Autónoma de Barcelona, Carlos III, Navarra, Santiago de Compostela, Sevilla, Rey Juan Carlos, San Jorge de Zaragoza y Vigo.

En cuanto a las universidades no españolas, destacan las iberoamericanas, pues 15 primeros o únicos firmantes proceden de ellas, lo que constituye el 10\% del total de trabajos analizados. Entre estas universidades sobresalen las argentinas, pues 7 autores pertenecen a universidades de este país sudamericano $(4,6 \%)$. La presencia de autores de universidades estadounidenses, asiáticas o europeas es residual, con 5 artículos en total, que suponen el 3,3\%.

m) Las diez universidades con más artículos

\begin{tabular}{|c|c|c|}
\hline Universidad & Artículos & Porcentaje \\
\hline Complutense de Madrid & 21 & 14 \\
\hline País Vasco & 9 & 6 \\
\hline Autónoma de Barcelona & 8 & 5,3 \\
\hline Carlos III & 8 & 5,3 \\
\hline Navarra & 8 & 5,3 \\
\hline Santiago de Compostela & 7 & 4,7 \\
\hline Sevilla & 5 & 3,3 \\
\hline Rey Juan Carlos & 5 & 3,3 \\
\hline San Jorge & 5 & 3,3 \\
\hline Vigo & 5 & 3,3 \\
\hline
\end{tabular}

En aquellos casos en los que los autores son profesores, resulta interesante conocer la facultad o centro al que están adscritos. La tabla "Facultad de los autores" muestra que, si bien en muchas ocasiones no se indica este dato, un 56,4\% de los primeros autores y el $44,4 \%$ de los segundos pertenecen a facultades de Comunicación, a facultades de Ciencias de la Información o a las que integran en el nombre el término comunicación como, por ejemplo, Facultad de Ciencias Sociales y de la Comunicación.

n) Facultad de los autores (en porcentaje)

\begin{tabular}{|c|c|c|}
\hline Facultad & Primer autor & Segundo autor \\
\hline Comunicación & 56,4 & 44,4 \\
\hline Filosofía y Letras & 4,3 & 0 \\
\hline Otras & 5 & 0 \\
\hline No se indica & 34,3 & 55,6 \\
\hline Total & 100 & 100 \\
\hline
\end{tabular}


Aunque en prácticamente la mitad de los casos no se ofrezca el dato, en el caso de los primeros firmantes es habitual que éstos posean el título de doctor $(39,6 \%)$ frente a una minoría de autores que no lo poseen $(8,1 \%)$. La tabla "Los autores son doctores" muestra asimismo que cuando se trata de los segundos firmantes, aumenta el porcentaje de no doctores $(22,7 \%)$ y disminuye el de quienes ostentan esta titulación $(27,3 \%)$. Esto último se debe a la tendencia de que aparezca como primer firmante el autor con un doctorado.

Pese a que también es un dato que no se indica en poco más del $25 \%$ de ocasiones, cuando el primer autor es doctor, lo es mayoritariamente en programas de doctorado de Comunicación (67,8\%). Este dato, junto al visto con anterioridad sobre la facultad de adscripción de los autores, muestra que la investigación en Historia de la Comunicación se desarrolla esencialmente en el ámbito científico de la Comunicación.

ñ) Los autores son doctores (en porcentaje)

\begin{tabular}{|c|c|c|}
\hline Doctor & Primer autor & Segundo autor \\
\hline Sí & 39,6 & 27,3 \\
\hline No & 8,1 & 22,7 \\
\hline No se indica & 52,3 & 50 \\
\hline Total & 100 & 100 \\
\hline
\end{tabular}

\section{Conclusiones}

La investigación sobre Historia de la Comunicación está presente, de manera estable y continua durante los diez años analizados, en las revistas seleccionadas como muestra, que son algunas de las más destacadas revistas españolas de Comunicación. Destaca Estudios sobre el Mensaje Periodístico como la revista que más cabida da entre sus páginas a los artículos objeto de estudio. Incluso se publican artículos de esta disciplina en aquellas revistas como Telos o Comunicar, centradas en ámbitos especializados que, a priori, están alejados de la dimensión histórica del fenómeno comunicativo.

Por lo que respecta al contenido de los artículos analizados, puede afirmarse que la Historia de la Comunicación sigue siendo predominantemente una Historia del Periodismo. También la Comunicación Audiovisual, incluyendo el cine, encuentra una notable presencia. En todas las revistas domina la prensa, de manera además muy rotunda en Estudios sobre el Mensaje Periodístico, excepto en Comunicar y en Telos, donde la Comunicación Audiovisual tiene la primacía. El medio más recurrente como objeto de investigación es la prensa escrita, seguida a bastante distancia por el cine y la televisión, lo que es coherente con el resultado expuesto anteriormente.

Por otro lado, la mayoría de artículos aborda el estudio del mensaje y del emisor en el proceso de la comunicación, mientras que las investigaciones sobre los receptores son muy minoritarias, si bien en Comunicar son las más habituales. Además, el enfo- 
que metodológico más utilizado para abordar el objeto de estudio es el cualitativo en todas las revistas, si bien Anàlisi, Comunicación y Sociedad, y Revista Latina de Comunicación Social son las que mayor entrada conceden a investigaciones cuantitativas.

Continuando con las conclusiones acerca del contenido, la Historia de la Comunicación es en buena medida una Historia Reciente de la Comunicación: el siglo XX concentra la inmensa mayoría de trabajos. Tan solo Anàlisi, Estudios sobre el Mensaje Periodístico y ZER publican trabajos centrados en los siglos XVI, XVII y XVIII. Del mismo modo puede afirmarse que es una Historia de la Comunicación en España, pues son escasos los estudios centrados en el extranjero. Únicamente los artículos publicados en Telos miran hacia otros países.

En cuanto a los autores, la mayor parte de los artículos tienen un solo firmante, caracterizado por ser profesor en una facultad de Comunicación de una universidad española y tener un doctorado en este ámbito.

\section{Bibliografía}

CASTILlO, A., y CARRETÓN, M. C. (2010). "Investigación en Comunicación. Estudio bibliométrico de las Revistas de Comunicación en España”. En: Comunicación y Sociedad, vol. 23, $\mathrm{n}^{\mathrm{o}}$ 2, p. 289-327.

CASTILLO, A., RUBIO, A., y ALMANSA, A. (2012). "La investigación en Comunicación. Análisis bibliométrico de las revistas de mayor impacto del ISI". En: Revista Latina de Comunicación Social, ${ }^{\circ}$ 67, p. 248-270.

COLLE, R. (2009): "La temática de Revista Latina de Comunicación Social, 19982008". En: Revista Latina de Comunicación Social, n 64, p. 71-85.

CHECA GODOY, A. (2008). Historia de la Comunicación: de la crónica a la disciplina cientifica. La Coruña: Netbiblo.

DE FILIPPO, D. (2013). "La producción científica española en Comunicación en WOS. Las revistas indexadas en SSCI (2007-12)". En: Comunicar, n 41, vol. 21, p. 25-34.

DE HARO DE SAN MATEO, M. V., y MARTÍNEZ MÉNDEZ, F. J. (2011). “Análisis temático de los artículos sobre Historia del Periodismo publicados en las revistas científicas españolas de Comunicación". En: Documentación de las Ciencias de la Información, vol. 34, p. 223-239.

DÍAZ NOCI, J. (2013). "A history of journalism on the internet: A state of the art and some methodological trends". En: Revista internacional de Historia de la Comunicación, $\mathrm{n}^{\mathrm{o}}$ 1, vol.1, p. 253-272.

ESCRIBÁ, D., y CORTIÑAS, S. (2013). "La internacionalización y las coautorías en las principales revistas científicas de Comunicación en España". En: Comunicar, $\mathrm{n}^{\circ}$ 41, vol. 21, p. 35-44.

GÓMEZ MOMPART, J. L. (2008). "Historia de la Comunicación e Historia del Periodismo: enfoques teóricos y metodologías para la investigación". En MARTÍ- 
NEZ NICOLÁS, M. Para investigar la comunicación. Propuestas teórico-metodológicas. Madrid: Tecnos.

JONES, D. E. (1998). "Investigación sobre comunicación en España. Evolución y perspectivas". En: Zer. Revista de estudios de comunicación, $\mathrm{n}^{\circ}$ 5, p. 13-51.

MARTINEZ NICOLÁS, M. (2008). "La investigación sobre comunicación en España. Evolución histórica y retos actuales". En MARTÍNEZ NICOLÁS, M. (coord.). Para investigar la comunicación. Propuestas teórico-metodológicas. Madrid: Tecnos, p. 13-52.

MARTÍNEZ NICOLÁS, M., y SAPERAS LAPIEDRA, E. (2011). "La investigación sobre Comunicación en España (1998-2007). Análisis de los artículos publicados en revistas científicas". En: Revista Latina de Comunicación Social, n 66, p. 101-129.

MONTERO DÍAZ, J., y RUEDA LAFFOND, J. C. (2001). Introducción a la Historia de la Comunicación Social. Barcelona: Ariel Comunicación.

MORAGAS I SPÀ, M. (1988). "Los estudios sobre comunicación y nuevas tecnologías en España: indicaciones sobre sus antecedentes y estado actual". En: Cuadernos de Investigación en Comunicación, $\mathrm{n}^{\mathrm{o}}$ 1, p. 11-19.

ROCA, D., y PUEYO, N. (2012). "La productividad científica en Comunicacióna través de la revista Zer". En: Revista Latina de Comunicación Social, $\mathrm{n}^{\mathrm{o}}$ 67, p. 292-321.

ROMÁN PORTAS, M. (2000). "Aspectos metodológicos de la historia de la comunicación”. En: Ámbitos, no 5, p. 119-128.

RUIZ ACOSTA, M. J. (1997). "La historia de la comunicación social en el ámbito universitario: objeto, fundamentos y problemas". En: Comunicación y Sociedad, vol. $10, \mathrm{n}^{\mathrm{o}} 2$, p.185-202.

SORIANO, J. (2008). "El efecto ANECA". En Actas y memoria final. Congreso internacional fundacional AEIC. Santiago de Compostela: Asociación Española de Investigación de la Comunicación.

XIFRA, J., y CASTILLO, A. (2006). "Forty years of doctoral public relations research in Spain: A quantitative study of dissertation contribution to theory development". En: Public Relations Review, no 32, p. 302-308.

VIGIL Y VÁZQUEZ, M. (1987). El periodismo enseñado. De la Escuela de "El Debate" a Ciencias de la Información. Barcelona: Mitre.

\section{El autor}

Ricardo Zugasti Azagra es Doctor en Comunicación por la Universidad de Navarra. Es profesor en la Universidad San Jorge de Zaragoza. Asimismo ha sido Visiting Research Fellow en la Universidad de Sheffield (Reino Unido) y profesor visitante en la Universidad del Istmo (Guatemala), en la Burgas Free University (Bulgaria) y en el Instituto Politécnico de Lisboa (Portugal). Sus ámbitos de interés investigador son la Historia de la Comunicación y la Comunicación Política. Es miembro del grupo de investigación "Medios de comunicación y campañas electorales", reconocido por el Gobierno de Aragón. 